\title{
Osteopontin Is Elevated during Neointima Formation in Rat Arteries and Is a Novel Component of Human Atherosclerotic Plaques
}

Cecilia M. Giachelli, Nancy Bae, Manuela Almeida, David T. Denhardt, “ Charles E. Alpers, and Stephen M. Schwartz Department of Pathology SJ-60 University of Washington School of Medicine, Seattle, Washington 98195; and *Nelson Biological Laboratories, Rutgers University, Piscataway, New Jersey 08855

\begin{abstract}
In an earlier report, we used differential cloning to identify genes that might be critical in controlling arterial neointima formation (Giachelli, C., N. Bae, D. Lombardi, M. Majesky, and S. Schwartz. 1991. Biochem. Biophys. Res. Commun. 177:867-873). In this study, we sequenced the complete cDNA and conclusively identified one of these genes, $2 \mathrm{B7}$, as rat osteopontin. Using immunochemistry and in situ hybridization, we found that medial smooth muscle cells (SMC) in uninjured arteries contained very low levels of osteopontin protein and mRNA. Injury to either the adult rat aorta or carotid artery using a balloon catheter initiated a qualitatively similar timedependent increase in both osteopontin protein and mRNA in arterial SMC. Expression was transient and highly localized to neointimal SMC during the proliferative and migratory phases of arterial injury, suggesting a possible role for osteopontin in these processes. In vitro, basic fibroblast growth factor (bFGF), transforming growth factor- $\beta$ (TGF- $\beta$ ), and angiotensin II (AII), all proteins implicated in the rat arterial injury response, elevated osteopontin expression in confluent vascular SMC. Finally, we found that osteopontin was a novel component of the human atherosclerotic plaque found most strikingly associated with calcified deposits. These data implicate osteopontin as a potentially important mediator of arterial neointima formation as well as dystrophic calcification that often accompanies this process. (J. Clin. Invest. 1993.92:1686-1696.) Key words: osteopontin • smooth muscle • angioplasty • neointima • atherosclerosis
\end{abstract}

\section{Introduction}

The most common characteristic of the response of blood vessels to injury is the formation of a neointima. This occurs following angioplasty, but is also seen in hypertension, renal and radiation injury, or trauma to any layer of the vessel wall (1). Most importantly, formation of the neointima following injury is believed to be an important model for the initial steps and progression of atherosclerotic lesions as well as the rapid restenotic changes seen after angioplasty $(2,3)$.

The steps involved in formation of the neointima have been

\footnotetext{
Address correspondence to Dr. Cecilia M. Giachelli, Department of Pathology (SJ-60), University of Washington, Seattle, WA 98195.

Received for publication 17 December 1992 and in revised form 7 May 1993
}

J. Clin. Invest.

(c) The American Society for Clinical Investigation, Inc.

$0021-9738 / 93 / 10 / 1686 / 11 \$ 2.00$

Volume 92, October 1993, 1686-1696 studied in detail, using the model of balloon angioplasty injury in the rat artery (2). In this model, endothelial denudation combines with medial damage to induce quiescent arterial smooth muscle cells (SMC) to enter the cell cycle, remodel their cytoskeletal components, and change adhesive properties and gene expression patterns. The net result is an activation or modulation of medial cells to become migratory and invade a normally SMC-deficient vascular compartment: the neointima. Once there, neointimal SMC adopt a number of special properties that distinguish them from the underlying medial SMC. We might, therefore, consider the cells that form the neointima as "activated" or modulated to perform the special functions associated with the repair process.

Although it is unclear what initiates SMC activation under these conditions, neointimal SMC share many of the properties of developmentally immature $\operatorname{SMC}(1,4)$ and it is intriguing to consider the possibility that similar mechanisms are used during development and during formation of the neointima. We have explored this hypothesis by differentially cloning genes that were selectively elevated in both rat neointimal and pup medial SMC compared to uninjured adult medial SMC $(5,6)$. As a result of this effort, we have discovered several genes which appear to mark an "activated" SMC phenotype both in vitro and in vivo. These included elastin and collagen $\mathrm{I}(\alpha 1)$ (6), as well as $2 \mathrm{~B} 7$, an mRNA that had not previously been described in smooth muscle cells (5). When partially sequenced, 2B7 showed high homology to osteopontin (secreted phosphoprotein 1) (5). In that report, we showed that 2B7 mRNA was present in total RNA prepared from the uninjured aorta and carotid arteries by Northern analysis. As predicted by our cloning strategy, 2B7 mRNA levels increased approximately fivefold in the carotid artery $48 \mathrm{~h}$ following de-endothelialization by balloon catheter, and remained elevated 7-14 d after injury (5).

In this paper we report full-length sequence data unequivocally identifying 2B7 as encoding rat osteopontin. Osteopontin is a highly acidic, secreted glycoprotein associated with bone morphogenesis $(7,8)$, cell transformation $(9,10)$, immune cell activation $(11,12)$, and bacterial resistance $(12,13)$. Although it is thought to play a role in bone mineralization and to mediate bone cell adhesion to calcified matrix via an arginineglycine-aspartate (RGD)-dependent mechanism (8), the function of osteopontin in other tissues is not yet understood. To this end, we have investigated the potential role of osteopontin in arterial tissue by determining its cellular distribution throughout the various phases of neointima formation after balloon catheter injury to rat aorta and carotid arteries. In ad-

1. Abbreviations used in this paper: AII, angiotensin II; GAPD, glyceraldehyde 3-phosphate dehydrogenase; RGD, arginine-glycine-aspartate: SMC, smooth muscle cells. 
dition, we report in vitro studies aimed at determining whether peptide factors thought to be important in arterial neointima formation regulate osteopontin mRNA and protein expression. Finally, we have found that osteopontin is a novel component of atherosclerotic plaque. Our data suggest that osteopontin may play an important role in arterial neointima formation.

\section{Methods}

Animal surgery and preparation of tissues. Adult male Sprague-Dawley rats weighing $\sim 400 \mathrm{~g}$ (at $\sim 3$ mo of age $)$ were purchased from either Simonsen Laboratories (Gilroy, CA) or Tyler Labs, Inc. (Bellevue, WA ). Rats were anesthetized with an intramuscular injection of Innovar-vet, $0.2 \mathrm{ml} / \mathrm{kg}$ and intraperitoneal injection of pentobarbital, 5.4 $\mathrm{mg} / \mathrm{kg}$ ( Pitman-Moore, Mundelein, IL). Angioplasty of the carotid or aorta was performed using a balloon embolectomy catheter as previously described (14). Briefly, the $2 \mathrm{~F}$ embolectomy catheter was introduced through the left external carotid, advanced into the abdominal aorta, inflated, and withdrawn. This sequence was repeated three times to achieve total endothelial denudation of the thoracic aorta and the left common carotid artery (as monitored by Evan's blue staining). The contralateral, uninjured carotid artery from the same animal, or aortas and kidneys from uninjured animals, served as control tissues. Surgically treated animals were housed individually in plastic cages and fed rat chow and water ad lib. At the appropriate times after injury, the rats were killed by ether inhalation, and, in some cases, tissue was perfusion-fixed in $4 \%$ paraformaldehyde, and embedded in paraffin as previously described (15). Microtome sections ( $5 \mu \mathrm{m}$ ) were mounted on vectabond-coated slides (Vector Labs, Burlingame, CA). Tissues prepared in this way from four to six rats per time point were used for both immunocytochemical and in situ hybridization studies. For isolation of RNA, aortas from four similarly treated rats were pooled and processed as previously described (5). Human atherosclerotic plaques were obtained from patients undergoing carotid endarterectomy surgery. Human coronary arteries were obtained after autopsy. These tissues were fixed and embedded as previously described (16).

Immunocytochemistry. The avidin-biotin peroxidase complex ( $\mathrm{ABC}$ ) technique as described by Hsu et al. (17) was used to detect the osteopontin epitopes in arterial tissue. The reagents for this analysis were purchased as a kit (Vectastain Elite; Vector Labs). Slides were deparaffinized, rehydrated, and incubated $35 \mathrm{~min}$ in $3 \% \mathrm{H}_{2} \mathrm{O}_{2}$ in methanol to block endogenous peroxidase activity. Nonspecific binding was blocked by incubating slides with normal rabbit or mouse serum in PBS containing $1 \%$ BSA and $20 \mu \mathrm{g} / \mathrm{ml} \beta$-galactosidase (Gibco-BRL Gaithersburg, MD). 2ArC and 2arN are affinity-purified, rabbit polyclonal antibodies that recognize the $\mathrm{COOH}$-terminal 73-214 amino acids and $\mathrm{NH}_{2}$-terminal 215-294 amino acids of mouse osteopontin fused to $\beta$-galactosidase, respectively (18). LF-7 is a previously characterized rabbit polyclonal antibody directed against human bone-derived osteopontin (19). These antibodies were used at 1:50 dilutions (for affinity-purified 2arC and $2 \mathrm{arN}$ ) and between 1:12,000 and 1:2,000 (for LF-7 antiserum). Control antibodies were used at equivalent concentrations and included: $(a)$ rabbit or mouse nonimmune serum; ( $b$ ) anti- $\beta$-galactosidase antiserum; and $(c)$ omission of the primary antibodies. Primary antibodies were diluted in PBS and incubated at room temperature for $1 \mathrm{~h}$. Washing and developing of color were performed as suggested by the manufacturer. For some studies with $\mathrm{LF}-7, \mathrm{NiCl}_{2}$ was added to the alkaline phosphatase substrate to generate a black reaction product, and counterstaining of sections was performed with methyl green. Cell types in human atherosclerotic plaque were distinguished using single label immunoperoxidase staining of serial sections with anti-CD68 and HAM-56, which recognize human macrophages (20), and HHF-35, which recognizes muscle actin and therefore is specific for smooth muscle in this context (21).
These antibodies were utilized as previously described (16). HAM-56 and HHF-35 were kindly provided by Dr. Allen Gown, University of Washington, anti-CD68 was purchased from DAKO (Copenhagen, Denmark ), and LF-7 was a gift from Dr. Larry Fisher, National Institutes of Health.

DNA sequencing. $2 \mathrm{~B} 7$ was cloned into pBluescript $\mathrm{S} / \mathrm{K}$ (Stratagene Inc. La Jolla, CA ) as previously described (5). Dideoxy sequencing was performed using a sequenase sequencing kit (IBI/Kodak Co., New Haven, CT) and vector or insert-specific oligonucleotide primers (Operon Technologies, Inc., Alameda, CA ). PCGENE and Intelligenetics Suite (IntelliGenetics, Inc., Palo Alto, CA) software was utilized for sequence comparisons. The sequence of nucleotides in $2 \mathrm{~B} 7$ was determined at least twice from both strands of the cDNA. Where differences were noted when compared to the sequence for rat bone osteopontin (22), a second SMC-derived osteopontin clone, 1B7, was also sequenced to rule out sequence errors due to cloning artifacts.

In situ hybridization. Riboprobe in situ hybridizations were performed on paraffin sections according to the method of Wilcox (15). The 2B7 plasmid was linearized with either BglI or MamI, and antisense or sense riboprobes were generated with $\mathrm{T} 7$ or $\mathrm{T} 3$ polymerase, respectively, in the presence of ${ }^{35}$ S-labeled UTP (DuPont \& Co., Boston, MA). Probes were separated from unincorporated precursors using a G-50 Nick column (Pharmacia LKB Biotechnology, Piscataway, $\mathrm{NJ})$. Peak radioactive fractions were pooled, precipitated in ethanol, and resuspended at $300,000 \mathrm{cpm} / \mu \mathrm{l}$. Hybridizations were performed at $52^{\circ} \mathrm{C}$ overnight. Slides were washed and autoradiographed as previously described (15). Exposure times were $14 \mathrm{~d}$ unless otherwise noted.

SMC culture, Northern, and Western blot analyses. Rat SMC were derived from normal adult or 12-d-old ( pup) rat aortic tunica media or 2-wk ballooned rat carotid neointima by enzymatic dispersion as previously described (23). Cells were grown in Waymouth's complete media (Gibco Laboratories, Grand Island, NY) supplemented with sodium pyruvate, nonessential amino acids and glutamate, $5 \%$ bovine serum (Hyclone Laboratories, Inc., Logan, UT), penicillin, and streptomycin, and used between passages 10 and 15 . Factors used for osteopontin mRNA and protein regulation studies were bovine pituitary bFGF, human PDGF (Collaborative Research, Inc., Bedford, MA), synthetic AII (Sigma Chemical Co., St. Louis, MO), porcine platelet TGF $\beta$ ( R \& D Systems, Minneapolis, MN), and FCS (Hyclone Laboratories, Inc.). Peptide dosages were chosen, based on published information such that maximal ligand-receptor interactions would be facilitated. RNA was isolated and analyzed by Northern analysis as previously described using the rat osteopontin cDNA clone, $2 \mathrm{~B} 7$ (5), the rat elastin cDNA clone, 56A3 (6), and a human glyceraldehyde-3-phosphate dehydrogenase clone (GAPD), pHcGap (24). Densitometry was performed using a densitometer (model R-112; Beckman Instruments, Inc., Fullerton, CA) on several different film exposures to ensure linearity of response.

For protein studies, cells were treated with the appropriate peptide factors and conditioned media were collected $48 \mathrm{~h}$ after addition. During this time there was no evidence of cell death as judged by light microscopy. Conditioned media samples were dialyzed against PBS, concentrated on centriprep ultrafiltration columns (Amicon Corp., Danvers, MA), and assessed for protein content using the Micro BCA protein assay kit with BSA as a standard (Pierce Chemical Co., Rockford, IL). Barium citrate adsorption was performed by adding $1 / 10 \mathrm{vol}$ $0.61 \mathrm{M}$ barium chloride and $1 / 10 \mathrm{vol} 0.13 \mathrm{M}$ sodium citrate to dialyzed conditioned media samples. The resulting precipitate and adherent proteins were washed three times with water and eluted into $0.2 \mathrm{M}$ sodium citrate. Equivalent amounts of protein were then dissolved in sample buffer, boiled, and applied to a $9 \%$ polyacrylamide gel by the method of Laemmli. Identical gels were either stained with Coomassie brilliant blue $\mathrm{G} 250$ or transferred to nitrocellulose using an electroblot system (Bio-Rad Laboratories, Richmond, CA ). Western blot analysis was performed using MPIIIB I0(1) $5 \mathrm{ng} / \mathrm{ml}$ and an alkaline phosphatase detection system. MPIIIB10(1) is a mouse monoclonal antibody directed against rat bone osteopontin obtained from the Developmen- 
tal Studies Hybridoma Bank (Iowa City, IA). In some cases, ${ }^{125} \mathrm{I}$-labeled sheep anti-mouse IgGF $\left(\mathrm{ab}^{\prime}\right)_{2}$ (NEN, Wilmington, DE) was utilized as the secondary antibody, and autoradiography was performed to detect radiolabeled bands.

\section{Results}

Complete sequence analysis of differential clone 2 B7. Complete sequencing of the 1,025-bp 2B7 cDNA and subsequent computer search indicated that this sequence shared $99.6 \%$ identity with the comparable region of the previously reported cDNA for rat bone osteopontin (22). Clone 2B7 begins at position 16 and ends at position 1040 relative to rat bone osteopontin mRNA and contains the entire coding region. Of the three base substitutions (out of 1,025 ), only one (a C to $\mathrm{T}$ transition at position 101) led to a conservative change in the amino acid sequence at position 8 from a Leu to Phe. The other differences in nucleotide sequence were an $A$ to $G$ substitution at position 160 and a $G$ to A substitution at position 529, neither of which changed the corresponding amino acid sequence.

Fig. 1 shows the amino acid sequence derived from translation of the largest open reading frame within the $2 \mathrm{~B} 7 \mathrm{cDNA}$ sequence. The rat smooth muscle-derived osteopontin mRNA encodes a 317 amino acid protein with several interesting features. These are indicated in Fig. 1 and include: (a) an $\mathrm{NH}_{2}$-terminal hydrophobic signal sequence typical of most secreted proteins; $(b)$ a highly acidic aspartate-rich domain potentially important for $\mathrm{Ca}^{++}$or cationic protein binding; (c) a putative

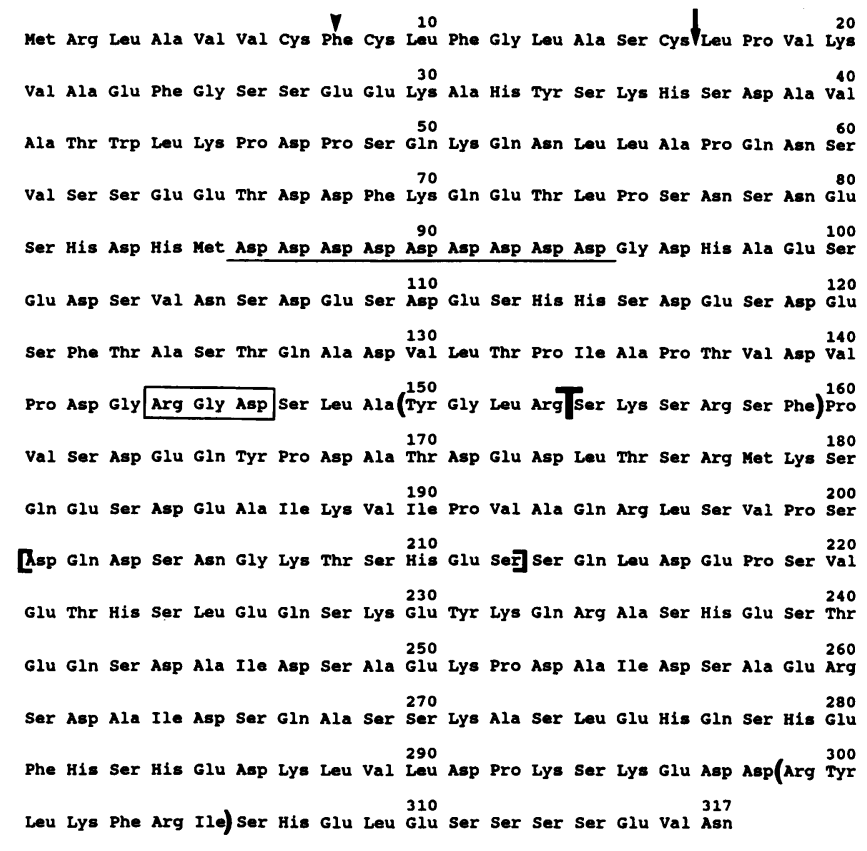

Figure 1. Amino acid sequence of rat smooth muscle-derived osteopontin produced from the $2 \mathrm{~B} 7 \mathrm{cDNA}$ nucleotide sequence. The arrowhead marks the single amino acid difference between rat smooth muscle and rat bone-derived osteopontin sequences. The vertical arrow indicates the putative signal peptide cleavage site. A highly acidic aspartate-rich domain is underlined. An RGD adhesion motif is boxed. Two potential heparin-binding sites as described by Prince (25) are shown in parentheses. A thrombin cleave site ( $T$ ) lies within one of these sites. A sequence showing homology to $\mathrm{a} \mathrm{Ca}^{++}$binding E-F hand is shown in brackets.
E-F hand-like $\mathrm{Ca}^{++}$binding site similar to that seen in thrombospondin and fibronectin; $(d)$ an RGD cell adhesion sequence common to many integrin ligands; $(e)$ a consensus sequence for thrombin cleavage (18); and $(f)$ two potential heparin binding sequences (25). These features are characteristic of osteopontin as described in a number of other species $(18,19,26)$.

In situ localization of osteopontin $m R N A$ and protein in normal rat arteries. As illustrated in Fig. $2 A$, uninjured rat arteries showed very low level staining with $2 \mathrm{arC}$, an affinitypurified, polyclonal antibody raised against the $\mathrm{COOH}$-terminal portion of mouse osteopontin expressed in Escherichia coli (18). The staining observed was diffuse in some areas, but some focal patches were also seen. In some sections, extracellular staining along elastic lamellae was evident (Fig. $2 A$, arrows). No staining was observed with control antibodies in any of the artery sections examined (not shown).

Likewise, osteopontin mRNA levels, as measured by in situ hybridization, were low in uninjured arteries (Fig. $3 A$ ). Signal value higher than that observed in arterial sections using the sense probe (not shown) was seen, however, in several cells scattered throughout the media in the carotid artery. This finding is in agreement with our previous Northern blot experiments showing osteopontin mRNA expression in carotid arteries (5). No antisense probe hybridization was observed in endothelial or adventitial cells. The sense probe gave no localized signal in any of the artery sections examined (not shown).

Increased osteopontin $m R N A$ and protein levels in rat neointimal SMC. $2 \mathrm{~d}$ after arterial balloon angioplasty, osteopontin mRNA levels were elevated in a subpopulation of carotid and aortic SMC closest to the lumen as shown in Fig. 3, B and $C$, respectively. $4 \mathrm{~d}$ after injury, SMC expressing osteopontin mRNA were still located at the luminal aspect, but cells adjacent to the external elastic lamina also showed elevated expression (Fig. $3 \mathrm{D}$ ). By $7 \mathrm{~d}$ after injury, the highest intensity of grains were over cells in the newly forming neointima, but a few cells at the media-adventitial interface still showed increased expression of osteopontin mRNA (Fig. $3 E$ ). $10 \mathrm{~d}$ after injury, all layers of neointimal SMC showed high level expression of osteopontin mRNA compared to the underlying media in both arteries (data not shown). By 3 wk after angioplasty, osteopontin mRNA had decreased substantially in the neointimal layer to levels equal to those in the underlying media in both the carotid (not shown) and aorta (Fig. $3 F$ ). However, a small population of neointimal cells directly adjacent to the lumen continued to show higher levels of osteopontin mRNA than neighboring intimal SMC. This localized expression of osteopontin mRNA was evident $6 \mathrm{wk}$ after injury, at which time osteopontin mRNA levels in all other parts of the vessel, including the remainder of the neointima, had decreased to uninjured levels (not shown). Corresponding sections probed with the sense probe showed no signal (shown in Fig. $3 G$ for $2-d$ ballooned aorta). At all time points, adjacent sections probed with the sense probe showed no specific signal (shown in Fig. $3 G$ for 2-d ballooned aorta).

Early after injury, osteopontin mRNA and protein levels correlated well in the different layers of the artery wall. $2 \mathrm{~d}$ after injury (not shown), only selected cells lining the lumen were positive for osteopontin protein. As shown in Fig. 2, $B$ and $C$, osteopontin protein was increased in the aorta and carotid $4 \mathrm{~d}$ after injury and was highest in the luminal SMC. These same regions were shown to have the highest levels of osteopontin 

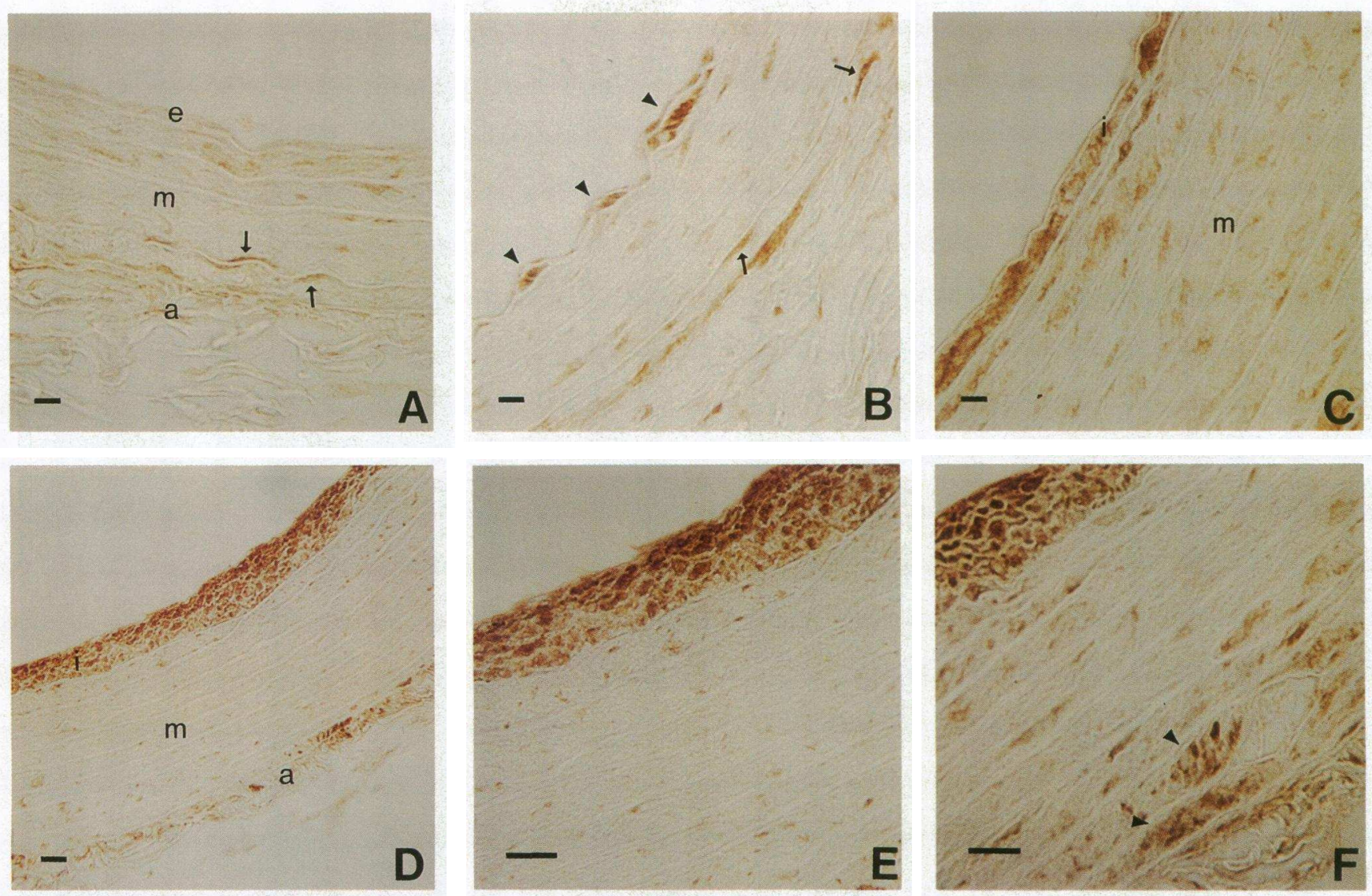

Figure 2. Immunohistochemical analysis of osteopontin protein levels in normal and balloon catheter-injured rat arteries. Aorta and carotid arteries from uninjured rats and rats subjected to balloon angioplasty were fixed, embedded, and stained for osteopontin using the affinity purified, 2arC antibody $(A-F) .(A)$ Uninjured rat carotid artery showing some localized patches of staining along the elastic lamellae (arrows); $(B)$ 4-d ballooned carotid showing three neointimal SMC (arrowheads) strongly positive for osteopontin, as well as discrete patches of staining along elastic lamella in the outer media (arrous); (C) 4-d ballooned aorta; (D-F) 3-wk ballooned aorta showing preferential osteopontin staining in the SMC of the neointima, as well as foci of positive cells in the outer media $(F$, arrowheads $)$. i, neointima; m, media; a, adventitia; $\mathrm{e}$, endothelium; bar, $10 \mu \mathrm{m}$.

mRNA early after injury (see Fig. $3, B-D$ ). Some sections prepared from 4-d ballooned rat aortas and carotid arteries also showed cells of the outer media expressing elevated levels of osteopontin similar to the mRNA pattern shown in Fig. $3 \mathrm{D}$. By $7 \mathrm{~d}$, the 2-3 cell layer-thick neointimas present in the aorta and carotid were highly enriched in osteopontin protein (data not shown), with a pattern identical to that observed for the mRNA (see Fig. $3 E$ ).

Antibody staining for osteopontin at 2 and $3 \mathrm{wk}$ after balloon angioplasty showed that the neointima was highly enriched for osteopontin (shown for $3 \mathrm{wk}$ in Fig. $2 \mathrm{D}$ ). The highest intensity of staining was cytoplasmic, but upon higher power examination (Fig. 2, $E$ and $F$ ) some staining could be seen extracellularly in distinct patches throughout the neointima and media over what appeared to be parts or edges of cells, or along elastic lamellae. Although staining was observed in selected cells of the media, the intensity was substantially lower than seen in the neointima. One exception was several clusters of SMC in the outer medial region whose level of osteopontin protein was nearly as high as that observed in the neointimal region (Fig. $2 F$, arrows). Counterstaining of these slides with hematoxylin showed that the labeled cells were within the ex- ternal elastic lamina, and not part of the adventitial layer (not shown).

The localization of osteopontin protein at this time was in striking contrast to the osteopontin mRNA pattern shown in Fig. $3 F$. Whereas osteopontin mRNA levels in the intima and media were decreased almost to uninjured levels, osteopontin protein levels were still high in the intima compared to the underlying media. The accumulation of osteopontin protein in cells of the intima, however, was transient. In sections prepared from arteries 4 and $6 \mathrm{wk}$ after injury, osteopontin protein levels had declined to uninjured levels in both the neointima and the media (data not shown). Thus, the difference in osteopontin mRNA and protein observed at $3 \mathrm{wk}$ most likely reflects the precursor-product relationship of the molecules. Identical staining patterns were observed when a second affinity-purified anti-mouse osteopontin antibody, $2 \operatorname{arN}(18)$, was used instead of 2arC to stain similar sections.

Regulation of osteopontin mRNA and secreted protein levels in cultured vascular SMC. To explore the regulation of osteopontin expression in SMC, postconfluent, quiescent SMC were treated with FCS, or several peptide factors thought to be important in neointima formation. As shown in Fig. 4, AII, 

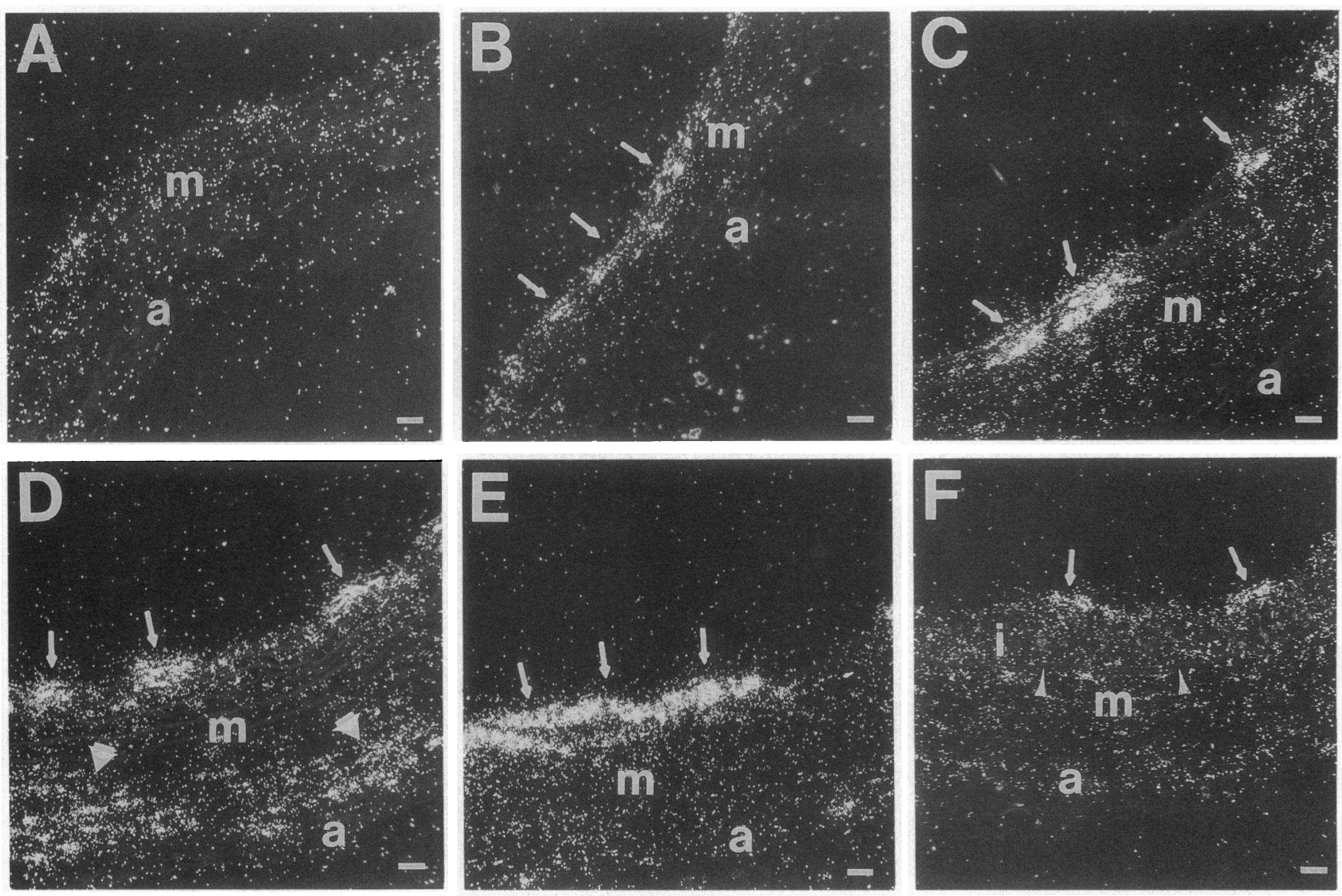

Figure 3. In situ hybridization analysis of osteopontin mRNA levels in normal and balloon catheter-injured rat arteries. Rat arterial sections were hybridized to the antisense $(A-F)$ riboprobes generated from transcription of the 2B7 plasmid. $(A)$ Uninjured carotid artery; $(B)$ 2-d ballooned carotid showing increased grains over cells adjacent to the lumen (arrows); $(C)$ 2-d ballooned aorta showing a similar pattern of high intensity signal localized to the luminal SMC (arrow's); (D) 4-d ballooned aorta showing high levels of grains over the luminal (arrows) and outer medial (arrow heads) SMC: $(E)$ 7-d ballooned aorta showing a 2-3 cell layer-thick accumulation of neointimal SMC (arrows) strongly positive for osteopontin mRNA: $(F)$ 3-wk ballooned aorta showing elevated grains over a subpopulation of neointimal cells closest to the lumen (arrows), but levels in the remainder of the intima $(i)$ similar to those in the underlying media $(m)$. Arrowheads point to the internal elastic lamina which separates the neointima and media: a, adventitia; i, neointima; m, media; bar, $10 \mu \mathrm{m}$.

bFGF, FCS, and TGF $\beta$ stimulated expression of osteopontin mRNA $24 \mathrm{~h}$ after treatment compared to untreated controls. FCS and AII appeared to be most effective, followed by TGF $\beta$ and bFGF. Osteopontin mRNA remained elevated in SMC 48 $\mathrm{h}$ after treatment with TGF $\beta$, bFGF, and AII, but not FCS. PDGF, on the other hand, had no effect on osteopontin mRNA levels at any time studied, despite the presence of both PDGF- $\alpha$ and $-\beta$ subunit mRNAs in these cells as measured by Northern blot analysis (Giachelli, C. M., unpublished observation).

Interestingly, osteopontin mRNA levels in untreated, postconfluent SMC were about twofold higher on day 3 than on days 0,1 , or 2 . This effect was specific to osteopontin, since elastin mRNA levels did not show the same pattern (Fig. $4 A$ ), and qualitatively similar results were obtained in three additional experiments. The reason for this elevation is unclear, but the data suggest that an endogenous regulator of osteopontin mRNA synthesis or degradation may be activated in postconfluent SMC. Further studies are required to test this possibility.

Western blot analysis was performed to determine whether
bFGF, AII, or TGF $\beta$ increased osteopontin protein released by SMC. A monoclonal antibody specific for rat bone osteopontin, MPIIIB10(1), was utilized since it was available in larger quantities than either of the rabbit polyclonal antibodies and thus could be used more conveniently for Western blotting. Fig. $5 \mathrm{~A}$ shows the specificity of MPIIIB10(1) antibody compared to 2arC for rat pup SMC-derived osteopontin. Both 2arC and MPIIIB10(1) detected a broad band at $66 \mathrm{kD}$ in rat pup-conditioned media, consistent with the size of osteopontin reported in rat bone (7). The identity of the $66-\mathrm{kD}$ band as osteopontin was further confirmed by its ability to be adsorbed to barium citrate and specifically eluted into $0.2 \mathrm{M}$ sodium citrate, properties previously reported for osteopontin (27). $2 \mathrm{arC}$ also interacted weakly with a smaller protein at $50 \mathrm{kD}$, which most likely represents a degradation product of osteopontin.

As shown in Fig. 5 B, osteopontin protein was increased in the media conditioned by confluent rat SMC after treatment with each of the three peptide factors. A major band at $66 \mathrm{kD}$, comparable to that seen for pup rat SMC, was present in 


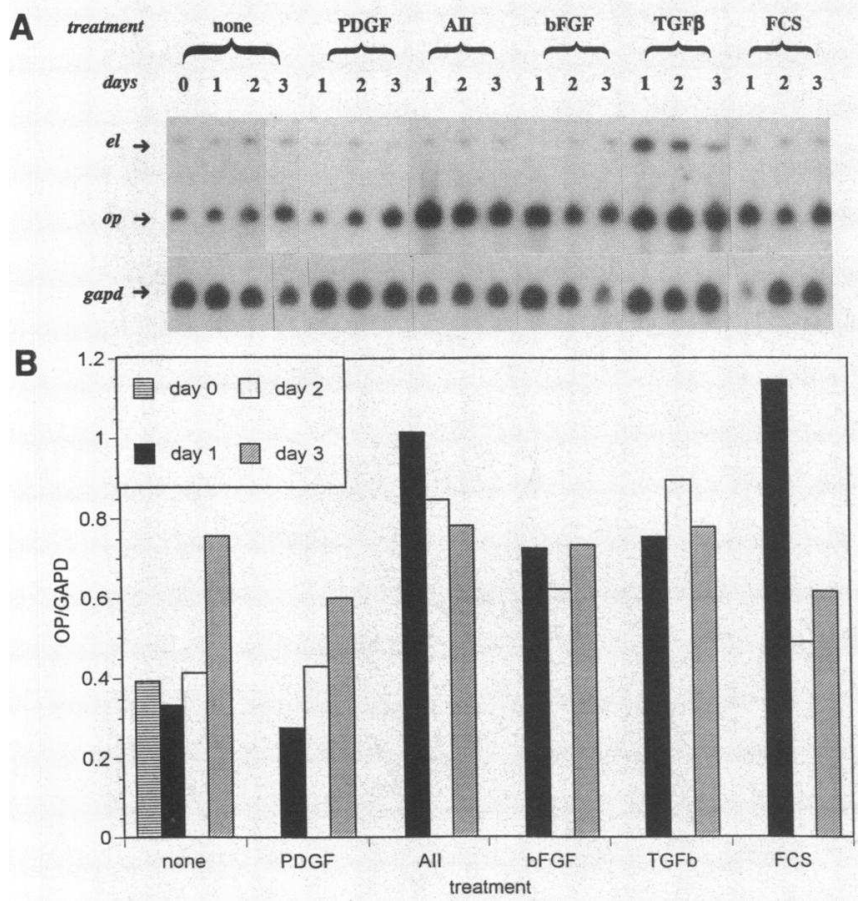

Figure 4. Effect of peptide factors and serum on osteopontin and elastin mRNA levels in cultured vascular SMC. $(A)$ Northern blot analysis of RNAs from SMC treated with PDGF $(10 \mathrm{ng} / \mathrm{ml})$, All $(10-6 \mathrm{M})$, bFGF $(10 \mathrm{ng} / \mathrm{ml})$, TGF $\beta(20 \mathrm{ng} / \mathrm{ml})$, or $20 \%$ FCS or left untreated (none). The autoradiogram shown resulted from a 24-h exposure of the hybridized filters. The sizes of the observed bands were estimated, based on migration of the 18 and $28 \mathrm{~S}$ ribosomal RNAs, and are as follows: elastin, $3.5 \mathrm{~kb}$; osteopontin, $1.6 \mathrm{~kb}$; and GAPD, $1.6 \mathrm{~kb}$. GAPD mRNA levels closely paralleled 28 and $18 \mathrm{~S}$ ribosomal RNA levels as judged by UV shadowing of the zetaprobe membrane (not shown). (B) Densitometric analysis of osteopontin mRNA levels shown in $A$ normalized to GAPD mRNA levels. Values are given as the ratio of OP to GAPD arbitrary densitometric units.

treated cultures compared to a very low level detectable in untreated SMC. Similar increases were observed when a second group of cells was treated identically and conditioned media proteins were examined $24 \mathrm{~h}$ after addition (not shown). The Coomassie staining patterns shown in Fig. $5 C$ indicated that equivalent amounts of protein were loaded onto the acrylamide gels used for Western blot analysis. Although these studies do not rule out changes in osteopontin protein levels in the culture media due to changes in degradation rate or compartmentalization, they strongly suggest that bFGF, AII, and TGF$\beta$ all increase synthesis and secretion of osteopontin in rat SMC.

Also shown in Fig. $5 \mathrm{~B}$ is a comparison of osteopontin protein levels found in the culture media of adult and pup rat SMC after $48 \mathrm{~h}$ of conditioning. As expected, based on previously reported mRNA levels (5), pup SMC-conditioned media contained a much higher steady-state level of osteopontin than comparably collected media conditioned by adult SMC. These levels were even higher than peptide factor-stimulated levels and suggest that pup SMC might be a good source of smooth muscle-derived osteopontin.

Osteopontin is a novel component of human atherosclerotic plaques. We examined 11 specimens of human artery ( 3 coronary arteries from autopsy and 8 carotid endarterectomy-de-

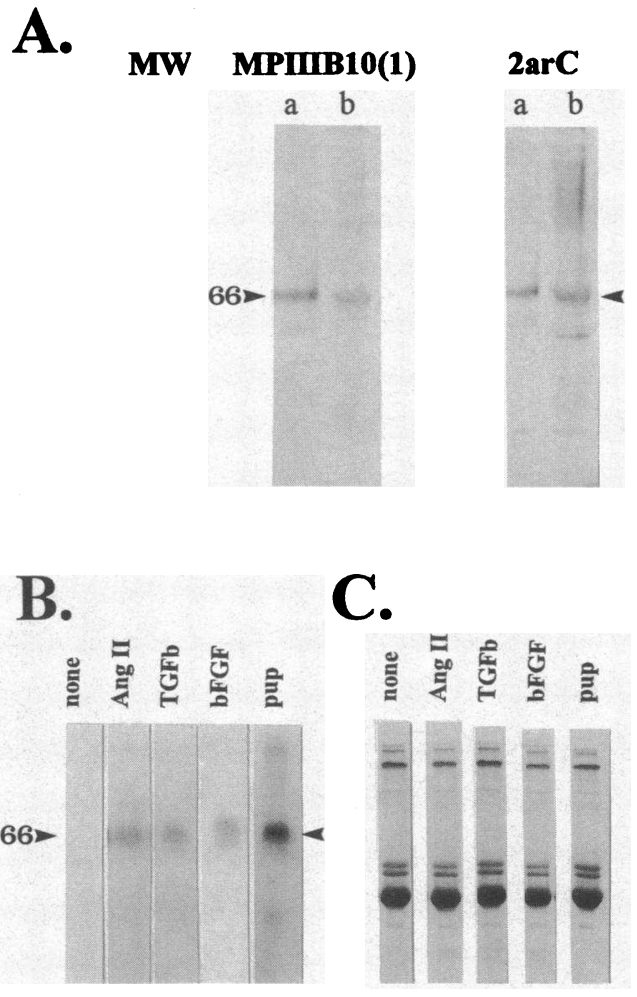

Figure 5. Western blot analyses of osteopontin released by rat vascular SMC in culture. $(A)$ Comparison of 2arC and MPIIIB10(1) antibodies. Rat pup SMC culture supernatants were partially purified by adsorption $(a)$ or dialyzed and concentrated $(b)$ and $25 \mu \mathrm{g}$ of the resulting proteins processed for Western blot analysis with either 2arC (1:50) or MPIIIB10(1) (1:5,000). MW, molecular weight markers, which were run concurrently. $(B)$ Effect of peptide factors on levels of osteopontin released by adult medial SMC. Adult medial SMC were treated identically to those described in Fig. 4 and the amount of osteopontin protein in conditioned media samples $2 \mathrm{~d}$ after treatment was determined by Western blot analysis using MPIIIB10(1) $(1: 5,000)$ as the primary antibody and ${ }^{125}$ I-labeled sheep anti-mouse IgG as the secondary antibody. The autoradiograms obtained after a 5-d exposure are shown. The apparent molecular weight of osteopontin estimated from concurrently run molecular weight markers is indicated in kilodaltons. $(C)$ Coomassie blue staining of the samples utilized for the Western blots of $B$, indicating equivalent protein loading for each lane.

rived samples) for osteopontin protein content by immunocytochemical staining using LF-7, an antibody that has been shown to be specific for human bone osteopontin (19). In arterial sections containing atherosclerotic plaques, LF-7 staining was focal and almost entirely limited to the intima as shown in Fig. $6 \mathrm{~A}$. While no staining was seen in the media of these vessels, faint cellular staining was observed in nerves and some scattered cells of the adventitia. Higher-power examination of the sections indicated that the LF-7 staining was associated with both cellular and acellular regions of the plaque. The strongest and most striking staining was observed surrounding basophilic calcified deposits, which often gave a granular appearance (Figs. $6 B$ and $7, A$ and $B$ ). In addition, the matrix surrounding cholesterol clefts showed elevated LF-7 staining compared to other matrix areas within the same plaque, or compared to normal samples (Figs. $6 B$ and 7, $A$ and $B$ ). Promi- 

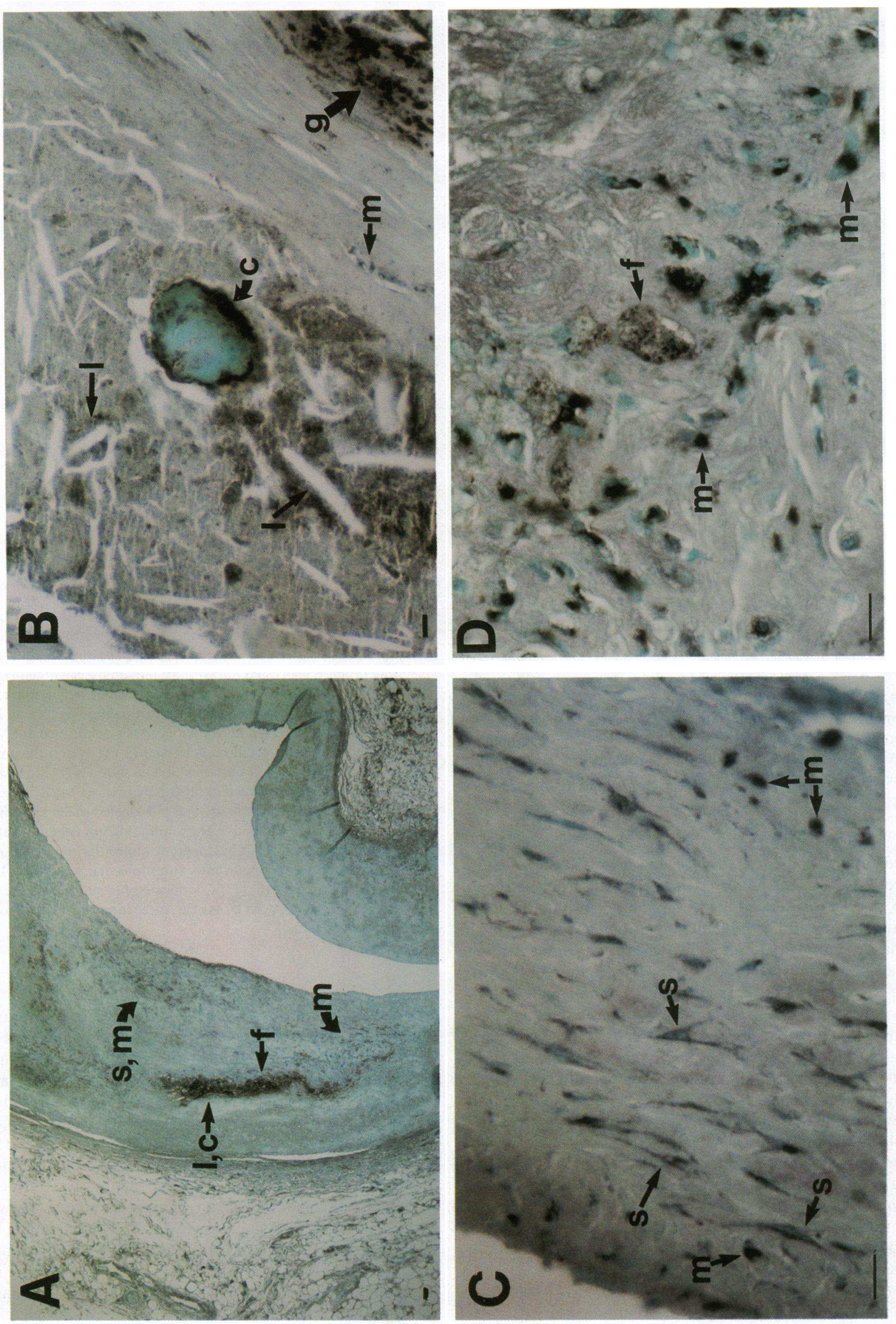
nent cellular LF-7 staining was observed in selected plaque SMC and macrophages, including macrophage-derived foam cells (Fig. 6, $C$ and $D$ ).

In two normal coronary arteries, no cellular LF-7 staining was observed in any of the vessel layers (not shown). However, occasional fine granular staining was observed in the matrix of the intima, but, in contrast to the plaque, these granules were not basophilic when an adjacent section was stained with hemotoxylin and eosin (not shown). The significance of this granular staining is unknown at present, but might represent early nidi for calcification or some other extracellular accumulation. These possibilities are currently under investigation.

\section{Discussion}

In a previous study, we used subtraction cloning to isolate genes that might be important in arterial neointima formation (5). In the present study, we used sequence analysis to conclusively show that one of these genes, $2 \mathrm{~B} 7$, encodes rat osteopontin. Furthermore, we have demonstrated by in situ techniques that osteopontin mRNA and protein are dramatically upregulated in vivo following rat arterial angioplasty selectively in neointimal SMC. Neointimal osteopontin expression was transient, occurring over the first $3 \mathrm{wk}$ after arterial injury. The striking temporal pattern and localization of osteopontin in nascent neointimal cells suggests that osteopontin may be important in the early processes regulating neointima formation in the rat such as migration, proliferation, and/or differentiation. Furthermore, we found significant osteopontin protein in human atherosclerotic plaques localized to selected SMC and macrophages, but most strikingly, in association with calcium deposits. No osteopontin protein was observed in normal human arterial media. Based on these findings, we speculate that osteopontin may be important in plaque progression as well as the dystrophic calcification commonly observed in these lesions.

Osteopontin is a secreted phosphoprotein originally described as a noncollagenous protein in bone matrix (8), but more recently observed in several nonmineralizing tissues, including kidney and arteries ( 5 ). In bone, osteopontin and other acidic phosphoproteins are thought to play critical roles in facilitating and regulating calcium deposition (28). Osteopontin is a low affinity, high capacity, calcium-binding protein (29), which can bind tightly to hydroxyapatite, the primary constituent of mineralized bone (22). In addition, osteopontin contains an RGD cell adhesion motif that is similar to that found in many cell-matrix adhesion molecules, including fibronectin, thrombospondin, and vitronectin (30), and it has been shown to interact with at least one integrin, the vitronectin receptor $\left(\alpha_{v} \beta_{3}\right)$ on osteoblasts (31). Based on these observations, as well as colocalization studies, it has been suggested that osteopontin may be critical for binding of osteoblasts and osteoclasts to the bone matrix (32). In addition, a role for osteopontin in cellular movement has been proposed, since osteopontin expression is upregulated in many tumor cell lines $(9,10)$ and correlates with metastatic potential (10). Furthermore, osteopontin (termed Eta-1 in that report) binds with high affinity to macrophages, and subcutaneous injection of purified Eta-1 into mice stimulates infiltration of macrophages to the site of injection (33).

The present study supports a role for osteopontin in facilitating cellular adhesion and/or migration during arterial neointima formation in the following ways. First, sequence analysis of $2 \mathrm{~B} 7$ verified that rat smooth muscle can make a form of osteopontin mRNA that includes an RGD-domain, previously shown to be important for cellular adhesion to osteopontin (22). Consistent with this, we have recently found that purified smooth muscle-derived osteopontin facilitates RGD-dependent adhesion and migration of rat vascular SMC in vitro (Liaw, L., and C. M. Giachelli, unpublished data). Second, in a rat model of neointima formation, osteopontin mRNA and protein levels were elevated specifically in SMC at the luminal surface of rat vessels at early times (between 2 and $7 \mathrm{~d}$ after angioplasty) when SMC leave the media and enter newly forming intima (2). Osteopontin levels were decreased to uninjured levels by 4 wk after injury, when SMC migration is at a minimum and the rate of intimal thickening has declined (2). Last, osteopontin protein was enriched in focal regions of human atherosclerotic intima and not found in the underlying media, providing evidence that gradients of osteopontin may exist in diseased vessels. Although correlative, these data suggest that at least one role for osteopontin in neointimal formation might be in facilitating cellular movement or preferential adhesion in the neointima. However, osteopontin might also be important in the proliferative or differentiation events that also occur in the early phases of neointima formation $(1,2)$. In this regard, osteopontin has recently been shown to correlate with cell cycle entry in cultured SMC (34). Development of specific in vivo inhibitors of osteopontin will help us greatly to distinguish between these possibilities.

Recent evidence has shown that growth factors play a role in initiating and regulating neointima formation following balloon angioplasty. Medial SMC proliferation has been shown to be due in large part to endogenous release of bFGF by the injured arterial media (35). Both bFGF and PDGF are thought to stimulate migration of the medial SMC into the neointima

Figure 6. Osteopontin is a novel component of human atherosclerotic plaques. $(A)$ Human atherosclerotic coronary artery section immunostained with anti-human osteopontin antibody, LF-7, and counterstained with methyl green. Arrows highlight areas of the plaque showing elevated LF-7 staining. These correlate with areas of lipid deposition $(L)$, calcium deposition $(C)$, macrophage $(M)$, SMC $(S)$, and foam cells $(F)$, as evidenced by eosin and hematoxylin staining and cell type-specific antibody staining performed on an adjacent section, as described in the Methods section (not shown). $(B-D)$. Human carotid endarterectomy samples immunostained with LF-7 and counterstained with methyl green. $(B)$ A region of plaque showing extensive calcium deposition $(C)$ embedded near a region of cholesterol and lipid accumulation $(L)$. Note the pronounced staining that outlines the calcified deposit and some cholesterol clefts, and exists throughout the surrounding matrix. At lower right are regions containing granular calcium deposits $(g)$. $(C)$ A region of plaque containing both SMC $(S)$ and macrophages $(M)$ that were LF-7-positive. Several striking examples of each cell type are indicated with arrows. Note that the pattern of staining in macrophages was polarized over a portion of the cell compared to SMC whose profiles were highlighted by the antibodies, thus allowing the visualization of the long processes of these cells. $(D)$ A region of plaque containing an inflammatory infiltrate. The cells in this region, including a prominent foam cell $(F)$, are strongly positive for LF-7 staining, showing polarization to one side of the cytoplasm. The LF-7 staining was contained in HAM-56and CD68-positive cells, as determined by staining of an adjacent section with these antibodies (not shown). Bar, $10 \mu \mathrm{M}$. 


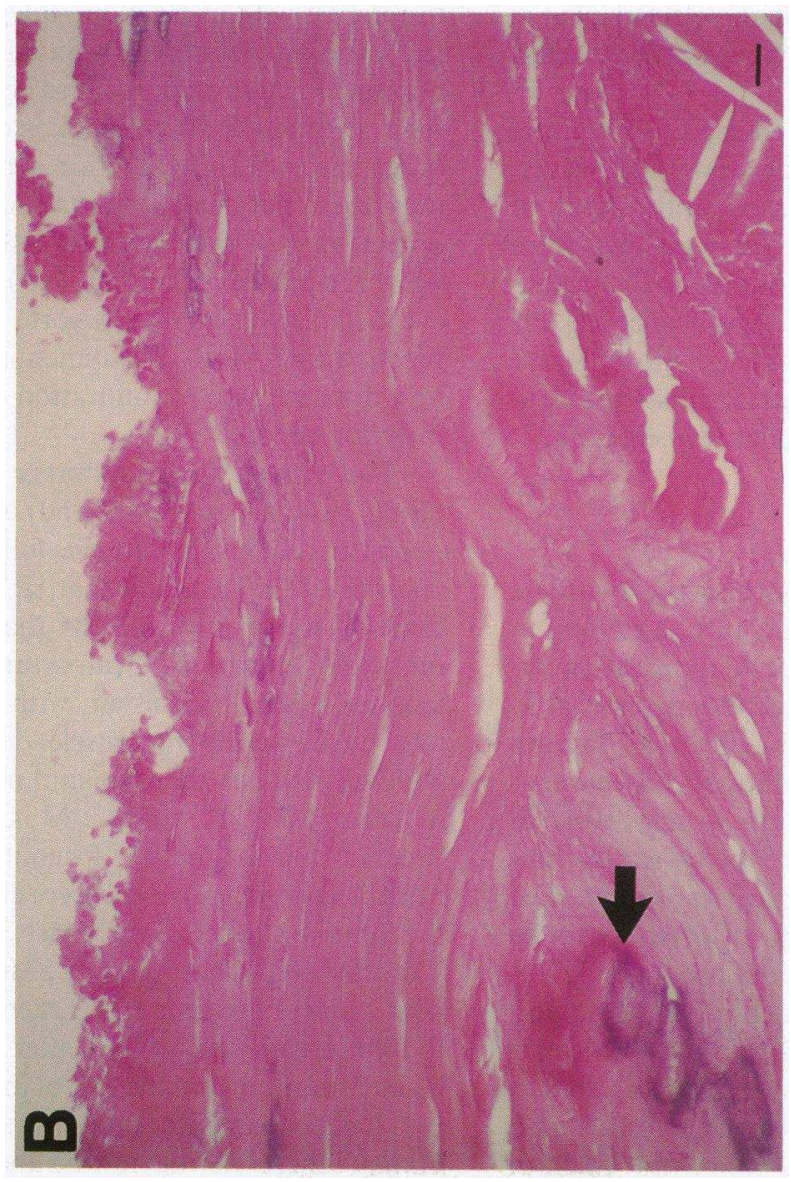

富

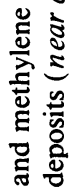

岕焉

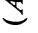

总

壱

兽

矛

艼

몬

응 융

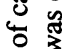

ํํำ

递贾

늘

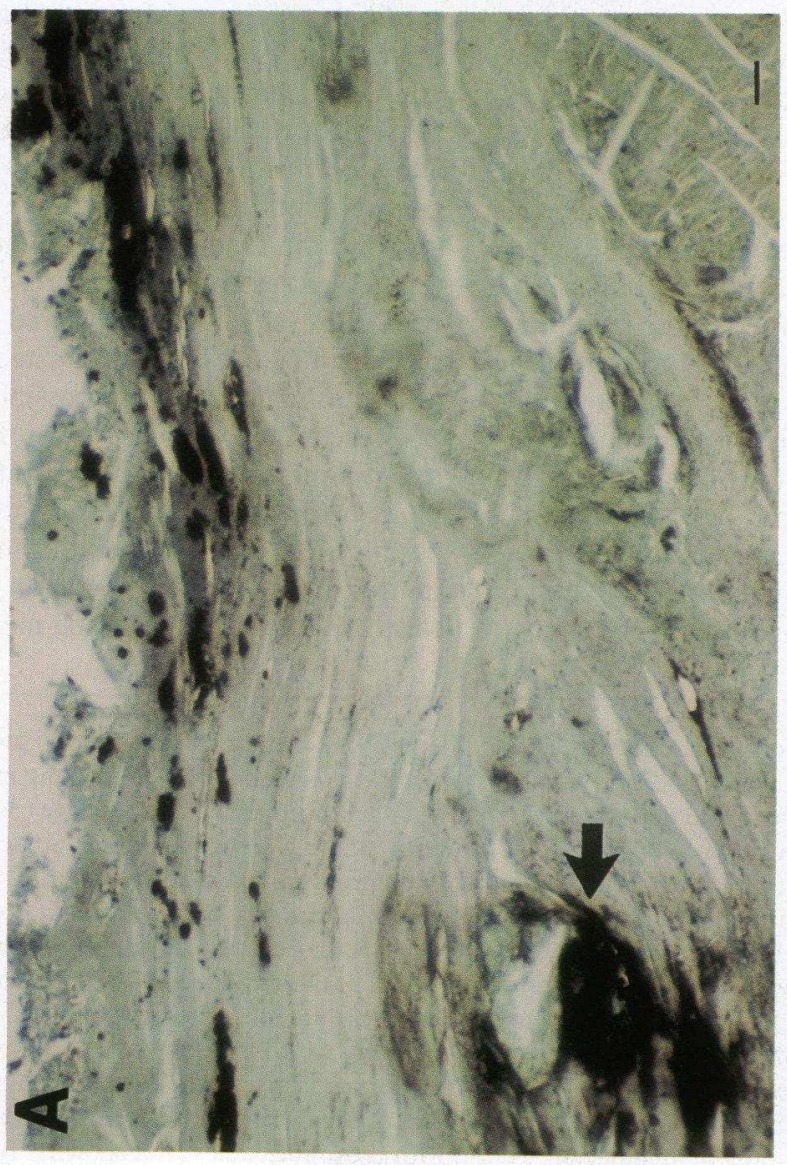

.

तु

然

핱

$\equiv$

突

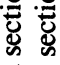

节 范

量氶

我

政

웡

할 을

就

需

过

过

卷

융 흉

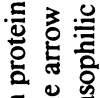

要

要

定.

ơ

西竞

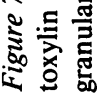


$(35,36)$. Factors initiating intimal proliferation are less well understood, but TGF $\beta$ has been shown to increase in arteries during wound repair (37), and both AII and TGF $\beta$ selectively stimulated neointimal SMC replication when given exogenously to animals in vivo $(14,37)$. Our in vitro studies suggest that several of these growth factors may also be important in regulating osteopontin expression in SMC. TGF $\beta$, AII, and bFGF, but not PDGF, elevated osteopontin mRNA and protein levels in confluent, quiescent adult medial aortic SMC. Although bFGF, TGF $\beta$, and PDGF have previously been shown to regulate osteopontin mRNA expression in other cell systems $(38,39)$, this is the first report of their action on vascular SMC. Furthermore, this is the first report of AII modulation of osteopontin mRNA levels in any cell type. Whether these factors are important in vivo regulators of osteopontin synthesis during neointima formation is currently being examined.

One of the most exciting findings resulting from this study was the focal osteopontin accumulation in human atherosclerotic plaques and its striking colocalization with calcium deposits. One possibility is that the osteopontin found in these areas was derived from plaque SMC and macrophages. Consistent with this idea, we observed intracellular osteopontin staining in both plaque SMC and macrophages (Fig. 6) and preliminary in situ hybridization studies indicate that these cell types synthesize osteopontin mRNA in human coronary atherectomy specimens (O'Brien, E., and C. M. Giachelli, unpublished data). Alternatively, osteopontin might be derived from the blood, since human plasma was shown to contain low levels of osteopontin protein (10). Regardless of the source, the close association of osteopontin protein with calcified regions, together with its biochemical properties ( 7 ) and proposed role in biomineralization (28), tempt us to speculate that osteopontin might also play a role in the calcification commonly observed in atherosclerotic lesions. Since calcification may contribute significantly to luminal occlusion and arterial dysfunction in atherosclerotic vessels (40), determining the possible roles of osteopontin in plaque formation and dystrophic calcification should be high priorities for further research.

\section{Acknowledgments}

The authors thank Dr. Larry Fisher for the gift of anti-human osteopontin antiserum, LF-7. We are indebted to Donna Lombardi and Marina Ferguson for help with in situ hybridization and immunohistochemistry, respectively. We also thank Drs. Earl Benditt, Edward O'Brien, Joan Lemire, Chuck Murry, and David Grainger for helpful discussions, and Holly Kabinoff for expert preparation of the manuscript.

This work was supported by NIH grants HL-03174 and HL-47151 and grant 3124 of the Council for Tobacco Research-USA.

\section{References}

1. Schwartz, S. M., R. L. Heimark, and M. W. Majesky. 1990. Developmental mechanisms underlying pathology of arteries. Physiol. Rev. 70:1177-1209.

2. Reidy, M. A., J. Fingerle, and M. W. Majesky. 1988. Proliferation of vascular smooth muscle cells in vivo. In Hyperlipidaemia and Atherosclerosis. K. E. Suckling and P. H. E. Groot, editors. Academic Press, London. 149-164.

3. Ross, R. 1988. The pathogenesis of atherosclerosis. In Heart Disease: A Textbook of Cardiovascular Medicine. 3rd ed. E. Braunwald, editor. W.B. Saunders Co., Philadelphia. 1135-1152.

4. Majesky, M. W., and S. M. Schwartz. 1990. Smooth muscle diversity in arterial wound repair. Toxicol. Pathol. 18:554-559.
5. Giachelli, C., N. Bae. D. Lombardi, M. Majesky, and S. Schwartz. 1991. Molecular cloning and characterization of 2 B7, a rat mRNA which distinguishes smooth muscle cell phenotypes in vitro and is identical to osteopontin (secreted phosphoprotein I, 2ar). Biochem. Biophys. Res. Commun. 177:867-873.

6. Majesky, M. W., C. M. Giachelli, M. A. Reidy, and S. M. Schwartz. 1992. Rat carotid neointimal smooth muscle cells re-express a developmentally regulated mRNA phenotype during repair of arterial injury. Circ. Res. 71:759-768.

7. Butler, W. T. 1989. The nature and significance of osteopontin. Connect. Tissue Res. 23:123-136.

8. Heinegard. D., K. Hultenby, A. Oldberg. F. Reinholt. and M. Wendel. 1989. Macromolecules in bone matrix. Connect. Tissue Res. 21:3-11.

9. Craig. A. M.. M. Nemir, B. B. Mukherjee. A. F. Chambers, and D. T. Denhardt. 1988. Identification of the major phosphoprotein secreted by many rodent cell lines as 2ar/osteopontin: Enhanced expression in H-ras-transformed 3T3 cells. Biochem. Biophys. Res. Commun. 157:166-173.

10. Senger, D. R., C. A. Perruzzi, and A. Papadopoulos. 1989. Elevated expression of secreted phosphoprotein I (osteopontin, 2ar) as a consequence of neoplastic transformation. Anticancer Res. 9:1291-1299.

11. Lampe, M. A., R. Patarca, M. V. Iregui, and H. Cantor. 1991. Polyclonal B cell activation by the Eta-1 cytokine and the development of systemic autoimmune disease. J. Immunol. 147:2902-2906.

12. Patarca, R., G. J. Freeman, R. P. Singh. F-Y. Wei, T. Durfee, F. Blattner, D. C. Regnier, C. A. Kozak, B. A. Mock, H. C. Morse III et al. 1989. Structural and functional studies of the early $\mathrm{T}$ lymphocyte activation I (Eta-1) Gene: Definition of a novel T cell-dependent response associated with genetic resistance to bacterial infection. J. Exp. Med. 170:145-161.

13. Fet, V., M. E. Dickinson, and B. L. Hogan. 1989. Localization of the mouse gene for secreted phosphoprotein 1 (Spp-1) (2ar, osteopontin, bone sialoprotein $1,44-\mathrm{kDa}$ bone phosphoprotein, tumor-secreted phosphoprotein) to chromosome 5. closely linked to Ric (Rickettsia resistance). Genomics. 5:375377.

14. Daemen. M. J. A. P., D. M. Lombardi, F. T. Boxman, and S. M. Schwartz. 1991. Angiotensin II induces smooth muscle cell proliferation in the normal and injured arterial wall. Circ. Res. 68:450-456.

15. Wilcox, J. N., K. M. Smith. L. T. Williams, S. M. Schwartz, and D. Gordon. 1988. Platelet-derived growth factor mRNA detection in human atherosclerotic plaques by in situ hybridization. J. Clin. Invest. 82:1134-1143.

16. O'Brien. K. D., D. Gordon, S. Deeb. M. Ferguson, and A. Chait. 1992. Lipoprotein lipase is synthesized by macrophage-derived foam cells in human coronary atherosclerotic plaques. J. Clin. Invest. 89:1544-1550.

17. Hsu, S. M., L. Raine, and H. Fanger. 1981. The use of avidin-biotin-peroxidase complex $(A B C)$ in immunoperoxidase techniques: A comparison between $\mathrm{ABC}$ and unlabeled antibody (PAP) procedures. J. Histochem. Citochem. 29:577.

18. Craig. A. M., J. H. Smith, and D. T. Denhardt. 1989. Osteopontin, a transformation-associated cell adhesion phosphoprotein, is induced by $12-\mathrm{O}$-tetradecanoylphorbol 13-acetate in mouse epidermis. J. Biol. Chem. 264:96829689.

19. Young, M. F., J. M. Kerr. J. D. Termine, U. M. Wewer, M. GeWang. O. W. McBride, and L. W. Fisher. 1990. cDNA cloning, mRNA distribution and heterogeneity, chromosomal location, and RFLP analysis of human osteopontin (OPN). Genomics. 7:491-502.

20. Gown, A. M.. T. Tsukada, and R. Ross. 1986. Human atherosclerosis. II. Immunocytochemical analysis of the cellular composition of human atherosclerotic lesions. Am. J. Pathol. 125:191-207.

21. Tsukada. T., D. Tippens, D. Gordon, R. Ross, and A. M. Gown. 1987. HHF-35, a muscle-actin-specific monoclonal antibody. I. Immunocytochemical and biochemical characterization. Am. J. Pathol. 126:51-60.

22. Oldberg. A.. A. Franzen, and D. Heinegard. 1986. Cloning and sequence analysis of rat bone sialoprotein (osteopontin) cDNA reveals an Arg-Gly-Asp cell-binding sequence. Proc. Natl. Acad. Sci. USA. 83:8819-8823.

23. Giachelli. C. M., M. W. Majesky, and S. M. Schwartz. 1991. Developmentally regulated cytochrome P450IAl expression in cultured rat vascular smooth muscle cells. J. Biol. Chem. 266:3981-3986.

24. Tso, J. Y.. X-H. Sun. T-H. Kao, K. S. Reece, and R. Wu. 1985. Isolation and characterization of rat and human glyceraldehyde-3-phosphate dehydrogenase cDNAs: Genomic complexity and molecular evolution of the gene. Nucleic Acids Res. 13:2485.

25. Prince, C. W. 1989. Secondary structure predictions for rat osteopontin. Connect. Tissue Res. 21:15-20.

26. Kerr. J. M., J. D. Fisher, and M. Young. 1991. The cDNA cloning and RNA distribution of bovine osteopontin. Gene (Amst.). 108:237-243.

27. Senger, D. R., C. A. Perruzzi, A. Papadopoulos, and D. G. Tenen. 1989. Purification of a human milk protein closely similar to tumor-secreted phosphoproteins and osteopontin. Biochim. Biophys. Acta. 996:43-48.

28. Gorski, J. P. 1992. Acidic phosphoproteins from bone matrix: A structural rationalization of their role in biomineralization. Calcif. Tissue Int. 50:391-396.

29. Chen, Y., B. S. Bal, and J. P. Gorski. 1992. Calcium and collagen binding properties of osteopontin, bone sialoprotein, and bone acidic glycoprotein 75 from bone. J. Biol. Chem. 267:24871-24878. 
30. Ruoslahti, E., and M. D. Pierschbacher. 1987. New perspectives in cell adhesion: RGD and integrins. Science (Wash. DC). 238:491-497.

31. Oldberg, A., A. Franzen, D. Heinegard, M. Pierschbacher, and E. Ruoslahti. 1988. Identification of a bone sialoprotein receptor in osteosarcoma cells. $J$. Biol. Chem. 263:19433-19436.

32. Reinholt, F. P., K. Hultenby, A. Oldberg, and D. Heinegard. 1990. Osteopontin-a possible anchor of osteoclasts to bone. Proc. Natl. Acad. Sci. USA 87:4473-4475.

33. Singh, R. P., R. Patarca, J. Schwartz, P. Singh, and H. Cantor. 1990. Definition of a specific interaction between the early $\mathrm{T}$ lymphocyte activation (Eta-1) protein and murine macrophages in vitro and its effect upon macrophages in vivo. J. Exp. Med. 171:1931-1942.

34. Gadeau, A-P., M. Campan, D. Millet, T. Candresse, and C. Desgranges. 1992. Osteopontin overexpression is associated with arterial smooth muscle cell proliferation in vitro. Arterioscler. Thromb. 13:120-125.

35. Lindner, V., R. A. Majack, and M. A. Reidy. 1990. Basic fibroblast growth factor stimulates endothelial regrowth and proliferation in denuded arteries. $J$. Clin. Invest. 85:2004-2008.
36. Jawien, A., D. F. Bowen-Pope, V. Lindner, S. M. Schwartz, and A. W. Clowes. 1992. Platelet-derived growth factor promotes smooth muscle migration and intimal thickening in a rat model of balloon angioplasty. J. Clin. Invest. 89:507-511.

37. Majesky, M. W., V. Lindner, D. R. Twardzik, S. M. Schwartz, and M. A Reidy. 1991. Production of transforming growth factor $\beta_{1}$ during repair of arterial injury. J. Clin. Invest. 88:904-910.

38. Noda, M., and G. A. Rodan. 1989. Type $\beta$ transforming growth factor regulates expression of genes encoding bone matrix proteins. Connect. Tissue Res. 21:71-75.

39. Rodan, S. B., G. Wesolowski, K. Yoon, and G. A. Rodan. 1989. Opposing effects of fibroblast growth factor and pertussis toxin on alkaline phosphatase, osteopontin, osteocalcin, and type I collagen mRNA levels in ROS 17/2.8 cells. J. Biol. Chem. 264:19934-19941.

40. Fleckenstein-Grun, G., and A. Fleckenstein. 1991. Calcium-A neglected key factor in arteriosclerosis. The pathogenic role of arterial calcium overload and its prevention by calcium antagonists. Ann. Med. 23:589-599. 